\title{
AREVA Developments for an Efficient and Reliable use of Monte Carlo codes for Radiation Transport Applications
}

\author{
Nicolas Chapoutier ${ }^{1,{ }^{*}}$, François Mollier ${ }^{2}$, Guillaume Nolin ${ }^{2}$, Matthieu Culioli ${ }^{2}$, Jean-Reynald Mace ${ }^{3}$ \\ ${ }^{1}$ AREVA NP Inc, Solomon Pond Park, Marlborough, MA 01752 \\ ${ }^{2}$ AREVA NP, 10-12 Rue Juliette Recamier, 69456 LYON CEDEX 06 \\ ${ }^{3}$ AREVA, 1 Place Jean MILLIER, 92084 PARIS LA DEFENSE CEDEX
}

\begin{abstract}
In the context of the rising of Monte Carlo transport calculations for any kind of application, AREVA recently improved its suite of engineering tools in order to produce efficient Monte Carlo workflow. Monte Carlo codes, such as MCNP or TRIPOLI, are recognized as reference codes to deal with a large range of radiation transport problems. However the inherent drawbacks of theses codes - laboring input file creation and long computation time - contrast with the maturity of the treatment of the physical phenomena. The goals of the recent AREVA developments were to reach similar efficiency as other mature engineering sciences such as finite elements analyses (e.g. structural or fluid dynamics). Among the main objectives, the creation of a graphical user interface offering CAD tools for geometry creation and other graphical features dedicated to the radiation field (source definition, tally definition) has been reached. The computations times are drastically reduced compared to few years ago thanks to the use of massive parallel runs, and above all, the implementation of hybrid variance reduction technics. From now engineering teams are capable to deliver much more prompt support to any nuclear projects dealing with reactors or fuel cycle facilities from conceptual phase to decommissioning.
\end{abstract}

\section{Introduction}

In the context of the rising of Monte Carlo transport calculations for any kind of application, AREVA recently improved its suite of engineering tools in order to produce efficient Monte Carlo workflow. Monte Carlo codes, such as MCNP [1] or TRIPOLI [2], are recognized as reference codes to deal with a large range of radiation transport problems. However the inherent drawbacks of theses codes - laboring input file creation and long computation time - contrast with the maturity of the treatment of the physical phenomena. The goals of the recent AREVA developments were to reach similar efficiency as other mature engineering sciences such as finite elements analyses (structural or fluid dynamics for example). 3 axes of development are presented in this paper:

- VICTORIA: a versatile Graphical User Interface based on CAD (Computer-Aided Design) tools to address radiation protection solvers,

- Calculation runs speed up,

- Chaining of Monte Carlo results to other solvers and engineering fields.

\section{VICTORIA}

VICTORIA (for Various Instances CAD calculation TOol and Radiation-propagation Interface by AREVA) is a software dedicated to AREVA engineering teams to address input decks and the post-processing of radiation transport simulations (notably MCNP and TRIPOLI).

\subsection{Challenges of the VICTORIA software}

The original idea of the project came from the observation that radiation solvers do not embed enough efficient environments for engineering workflow. Indeed geometry creation and the definition of other simulation parameters have to be defined by an ASCII file based on keywords. Compared to other engineering sciences no aided engineering software permits to assist the user to efficiently perform analyses. Especially no general CAD integration is natively available. The modification of existing models can be complex and source of human errors. The primary author of a Monte Carlo model is often the only one capable to adapt it for other purpose or design modifications. These drawbacks do not meet the profitable requirements of the engineering projects. Indeed prompt iterations are expected to progress efficiently in interface with other disciplines.

Also the engineering teams are sometimes using different radiation protection solvers as point-kernel or Monte Carlo calculation codes (MCNP or TRIPOLI according to the project context). Despites their similarities each solver has its own syntax and specificities and a model built for one solver cannot be directly reused to another. In term of skill management,

Corresponding author: nicolas.chapoutier@areva.com 
keeping trained engineers for several solvers is also a challenge.

\subsection{Technology choices}

To fulfil the objectives of the VICTORIA software, AREVA based its developments on the ANSYS WORKBENCH platform. ANSYS, world leader for Computer Aided Engineering software, has a reliable experience of integrated simulation system. The ANSYS WORKBENCH platform gathers finite elements solvers (structural analyses, computational fluid dynamics (CFD) for example) with a common and compatible data models management. Also $3 \mathrm{D}$ modelling software is included to create geometries, edit, clean up or repair CAD imports.

The ACT (ANSYS Customization Toolkit) product recently released by ANSYS enables to add customization to the WORKBENCH platform. This application programming interface permits to redesign the purpose of the traditional ANSYS analyses for radiation protection solvers. Existing features: CAD modeling, meshing algorithm, 3D post-processing are directly inherited from the platform for customization. The specific developments in ACT can follow the future new releases of the ANSYS Workbench platform, meaning that all new improvements from ANSYS in the standard platform will be accessible by the VICTORIA users. In other words VICTORIA is not a standalone application and its sustainability can be ensured.

\subsection{Overview of VICTORIA}

\subsubsection{VICTORIA Workflow}

The creation of a radiation protection analyses is based on a sequence of several steps:

- Material database creation,

- 3D geometry importation and / or creation,

- Model: material assignment to bodies,

- Setup: simulations parameters (particles type, sources and tally creation),

- Solution and results: including post-processing features: import of output files for $2 \mathrm{D} / 3 \mathrm{D}$ rendering of tallies.

Each workflow defined in a project is pictured on the project schematic front page. The project schematic offers a comprehensive and visual overview of the cases dealt. Duplication and modification of whole workflow can be done. The saved projects stored all the needed information ensuring traceability and reliable management of the models.

As an overview, in the figure 1, 2 MCNP analyses are run. Both analyses shared the same material libraries and 2D geometry. The review of such studies where identical portions of the model are used for different cases is speeded up.

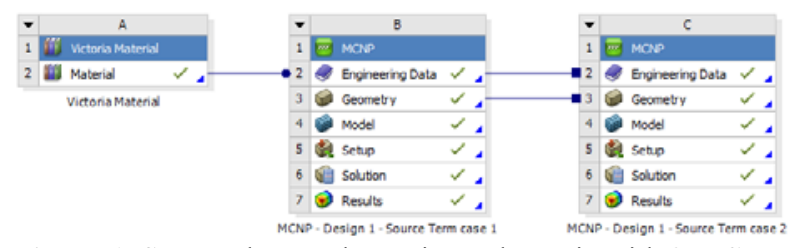

Figure 1. Screen shot on the project schematic with $2 \mathrm{MCNP}$ analyses

At any steps of the workflow definition the user can modify upstream parameters. For example, the modification of geometrical property (modification of a thickness, addition of parts...) will be automatically taken into account into the downstream parameters (update of material assignment, and source or tally properties for example). This feature is perfectly adapted for design phase. Indeed sensitivity analyses, or design modifications can be easily done. All the unchanged parameters will be inherited from the previous analysis. So the engineer on the basis of a workflow can efficiently study alternative designs.

Also as pictured on figure 2 , the user can convert workflow from one solver to another. This means that all objects (a material, a geometry, a source or a tally defined on a part of the model) can be addressed either in the MCNP or TRIPOLI syntax.

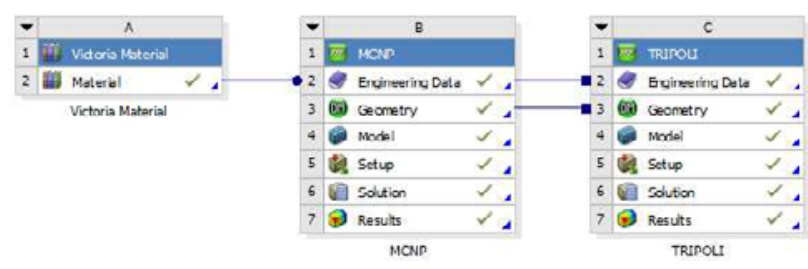

Figure 2. Screen shot on the project schematic-MCNP and TRIPOLI cases

Figure 2 shows a project with 2 analyses. Here the MCNP and TRIPOLI cases shared the same 3D geometry meaning that the geometry built is translated both in MCNP and TRIPOLI syntax.

In order to convert each objet of the workflow either in MCNP or TRIPOLI syntaxes each attributes have the capability to be addressed to the dedicated syntax. This bidirectional expression is doable because of the relative similarities in the two solvers. For a large majority of MCNP keywords there is its equivalent in the TRIPOLI syntax. If a specific attribute exists in solver without any equivalent in the other, the attributes is exposed in the Graphical User Interface (GUI) only in the frame of the adequate workflow and must be filled by the user. This is particularly notable for the simulation parameters where each solver has its own logic. In order to smooth the experience of the user default values are set corresponding to typical parameters for transport calculations. If needed these values can be redefined by the user thanks to the GUI.

\subsubsection{Material Database}

A dedicated GUI has been developed in order to create material databases useable for the radiation 
solvers. Composition (chemical distribution or Plutonium vectors for example) and density are defined by the user for each material. Routines are implemented in order to compute mass density from atomic concentration or vice versa. The mixture of material is also allowed with the automatic computation of the resulting density and composition. Temperature dependent cross section and thermal treatment options are also callable. Natural abundance of elements are stored to aid the material definition for neutron problems.

The dataset structure built to manage these databases allows the storage and the sharing between several users. This ensures traceability and quality.

\subsubsection{Geometry and CAD integration}

The surfaces allowed by Monte-Carlo solvers are reduced to basics shapes: planes, cylinders, sphere, cones, torus, ellipsoids, hyperboloid and paraboloid. Other surfaces as splines are discarded. The algorithm embedded within VICTORIA converting 3D bodies to Monte-Carlo geometry syntaxes deals with the main useful shapes: planes, sphere, cylinder, cone and torus. Each of these surfaces is also correctly written when coordinates transformations are applied (translation and/or rotation). In the case of torus expression for MCNP the transformations are limited, as allowed by the solver, to transformations parallels to the axis of the global system.

The geometry creation and preparation are the main time consuming step. As described above the geometry creation can be done by direct modeling and / or CAD or MCNP/TRIPOLI file import.

The challenge of the geometry preparation is to get a set of bodies whom the shapes comply with the surfaces allowed and the limitations of the conversion algorithm. This is particularly true when CAD files are used. Generally CAD files are not built for radiation purposes and may contains spline surfaces, complex bodies and irrelevant details for radiation aspect. It is required to limit the complexity of the bodies in terms of the number of surfaces and ambiguity surfaces involved (ambiguity surfaces are surfaces which are not part of the external boundaries of a solid but are needed to decompose spatially the body in a set of non-overlapping shapes). To achieve this difficulty, the user is assisted by several tools added to the 3D modeler such as:

- detection of unsupported surfaces,

- capability to replace a surface by a supported one,

- detection of unsupported bodies,

- spatial decomposition of complex bodies.

Also because the Monte Carlo geometry must cover any coordinate points of the system, the addition of internal or enclosure volumes inside and around equipment can be built in the $3 \mathrm{D}$ modeler. The same approach in term of preparation (spatial decomposition) is also required for these volumes.

Figure 3 presents how the CAD model of a steam generator is prepared by deleting useless details and unsupported surfaces (e.g. blended surfaces).

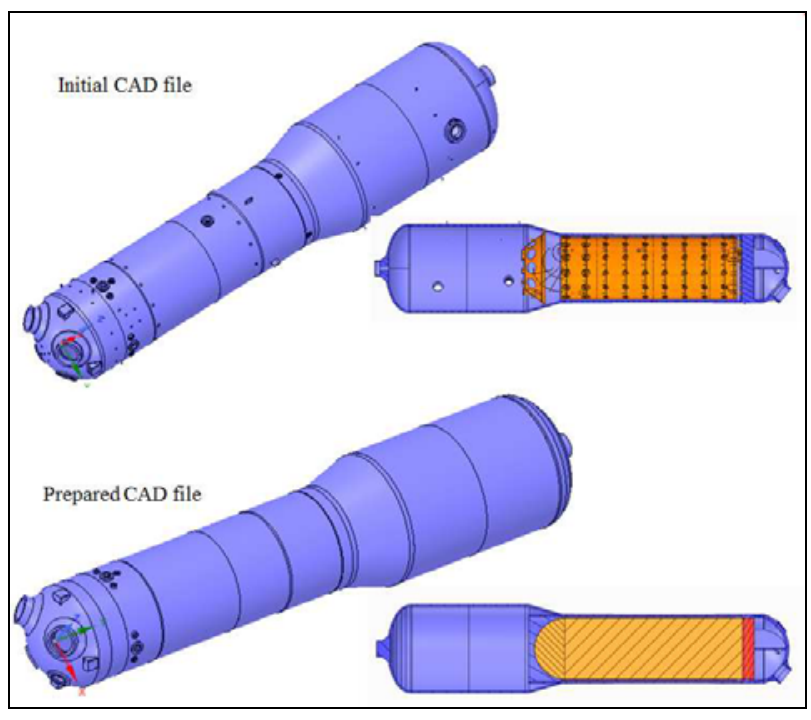

Figure 3. Example of geometry preparation

Tools have also been integrated to deal with lattice structures. A common add-in to the 3D modeler GUI is used to generate both MCNP and TRIPOLI lattices for Cartesian and hexagonal pattern.

VICTORIA developments also address the latest features of MCNP6. Notably MCNP6 permits to describe geometries with Unstructured Meshes. Instead of building the geometry based on traditional surfaces (or macro bodies), the bodies are defined as a set of tetrahedrons. This offers the capability to convert any kind of CAD shapes to MCNP6.

Figure 4 presents an extensive test case of this feature applied to model the entire quarter of a reactor building. More than 400000 tetrahedrons are used in this model. However such technic has inherent drawbacks as an increase of the computer runs of a factor of $\sim 10$ for equivalent configuration modeled with traditional surfaces. However this functionality prefigures what could be in a next future a more global approach to deal with CAD integration for Monte Carlo simulation and demonstrates the versatility of the software using mesh algorithms available in the ANSYS platform.

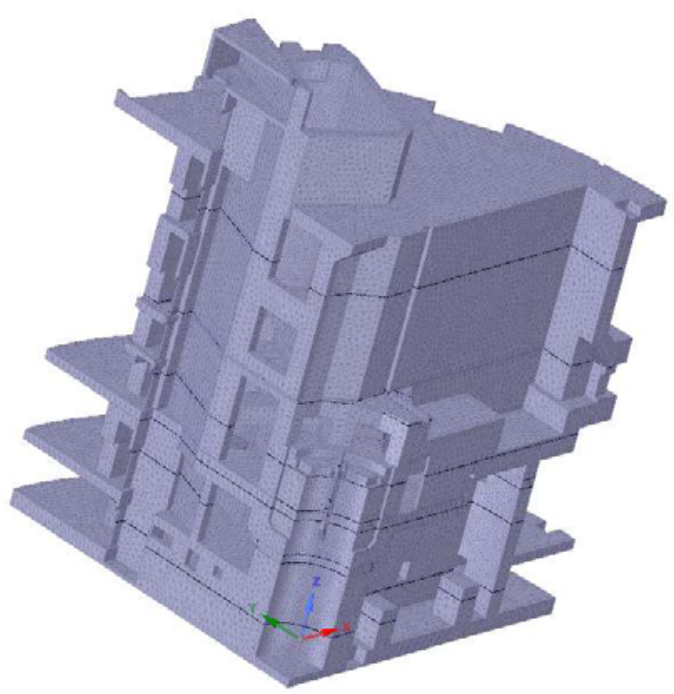

Figure 4. Example of MCNP6 unstructured meshes generates via VICTORIA 


\subsubsection{Model and setup definition}

In this step of the workflow the user can:

- assign the materials to volumes,

- set the simulation parameters (mode, termination criterion...),

- define the sources,

- define the tallies.

Figure 5 presents a view of a PWR model with material assignment.

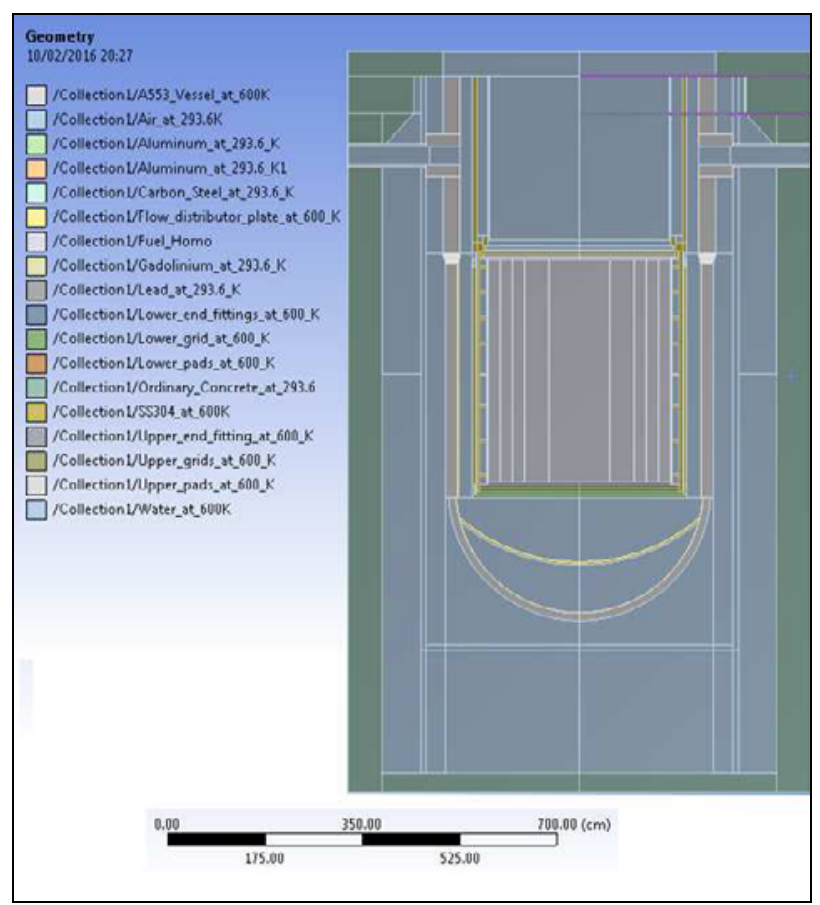

Figure 5. Example of a PWR model generated with VICTORIA

The sources can be defined for both neutron and gamma problems. The shape of sources can be a set of points, on surfaces or on volumes (cells rejection).

The surface sources definition is particularly used to study problems with contamination on systems. The locations of the sampling of source particles on the surfaces are determined by a specific routine.

For volume sources, the syntax generated is a combination of sampling volumes associated with cell rejection. The algorithm embedded within VICTORIA determines the shape and the size of these elementary volumes (box, cylinder or sphere) in order to limit the waste of computer time for cell rejection.

All types of tallies can be defined in the GUI: point, surface, cell tally and also meshed tallies: structured tallies (FMESH and TMESH cards of MCNP and extended mesh of TRIPOLI) and Unstructured Meshes (EMBEE card of MCNP6). Response functions (flux, dose, reaction rate) and energetic bins are also callable.

At the end of this step the solver input file is exported in a user defined directory. The solver run is not part of the VICTORIA workflow. The run is done according to the user preference (linux or windows) and the cluster availabilities.

\subsection{Post processing}

Post-processing features allows the parsing of solver output files to display 3D rendering and edit Excel sheets of results. Figure 6 is such an example of neutron mesh tally superimposed on the lower part of the reactor cavity pit. All the meshed tallies of the solvers (EXTENDED mesh for TRIPOLI and FMESH, TMESH and EMBEE for MCNP) can be displayed, 3D rendering capabilities are inherited from the mature finites elements tools (iso-lines, clipping, probe, etc...).

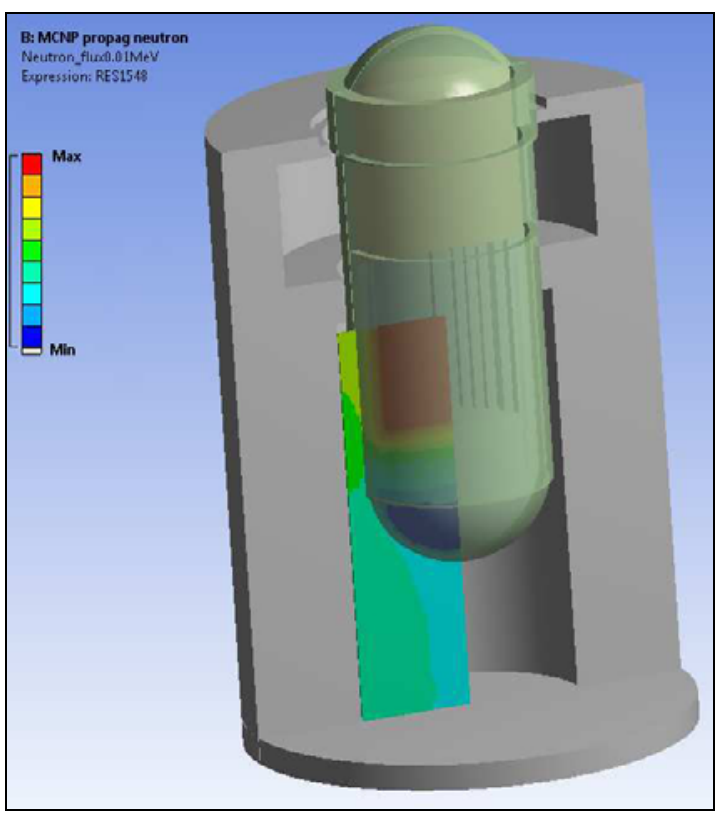

Figure 6. Example of 3D mesh tally rendering

The generation of CSV files or mesh based file format (VTK) is also commonly done to simplify the interface with other tools or engineering fields. This is discussed in section 4 .

\section{Acceleration of calculations}

Efforts have been done to decrease the computer running time of the calculations. In addition to the use of parallel runs, the use of hybrid variance reduction technics is deployed for MCNP usage. The term hybrid is used because of the methods imply the use of deterministic code prior to the Monte Carlo run. For transport calculation the CADIS method [3] is used to generate MCNP Weight Windows input files.

More precisely CADIS method is used to improve the streaming of particles at one specific location. In that case an adjoint calculation is run with a SN 3D deterministic code [4] on an equivalent model of the MCNP case. The Weight Windows values of the user defined mesh are inversely proportional to the adjoint flux distribution of a point source located at the target coordinate. 
For problems requiring to reach convergence criteria in several locations the Forward Weighted CADIS method is used. In that case a forward calculation is firstly run to assess the adequate strengths of the adjoint sources.

The FW-CADIS method has demonstrated its efficiently on a large set of study case both from neutron and gamma for several applications (shielding, fluence, energy deposition, activation). The figure below shows an example of fast fluence calculation on a PWR model. In that case, the suggested area of convergence is the entire model (from the fuel active part to the concrete cavity pit). Both traditional and extended beltline area are calculated simultaneously. For this demonstrative case the size of the mesh tally voxel is $8 \times 8 \times 19 \mathrm{~cm}^{3}$. On that example the fast fluence in the extended area (nozzle region) is calculated with a relative error of $2 \%$ after 2 hours of calculation and $0.7 \%$ after 18 hours of calculation with 7 tasks at $2.93 \mathrm{GHz}$.

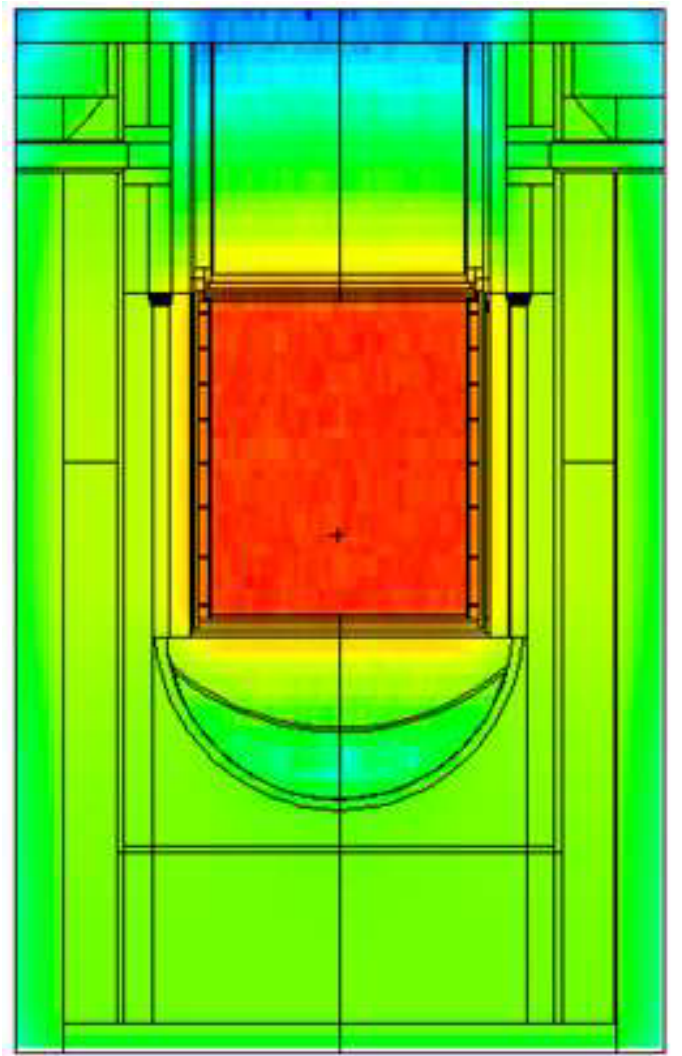

Figure 7. Example of MCNP fluence calculations on extended beltline area

Developments have also been done in order to run CADIS methods on Unstructured Mesh syntax of MCNP6. On figure 8, the FW-CADIS method has been applied to the model presented on figure 4 in order to calculate the neutron flux distribution in the concrete civil work. Reaching simultaneously convergence criteria in several locations is particularly useful for activation analyses.

Hybrid technics offer a global approach and ready to use for the engineering teams. The main difficulties remain on the choice of an appropriate mesh especially while dealing with small penetrations.

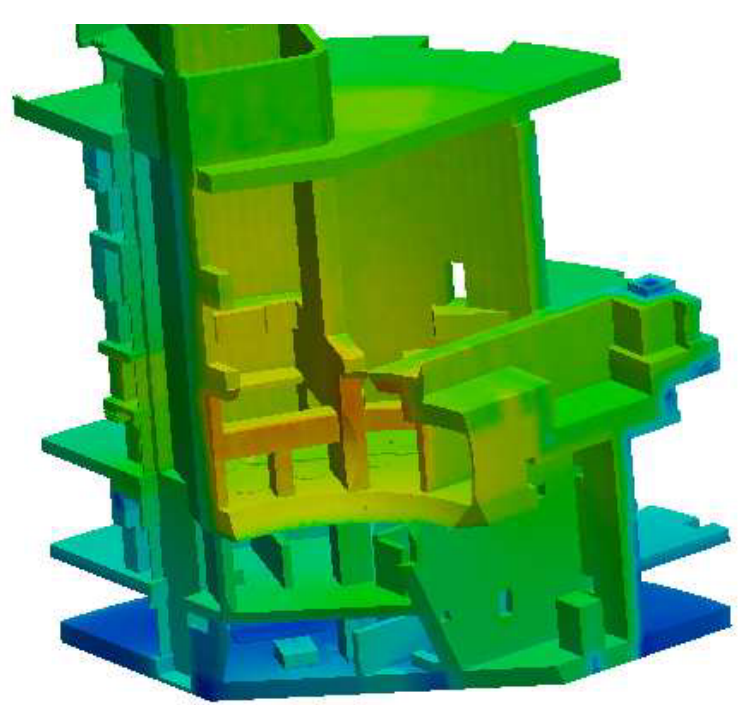

Figure 8. Example of the calculation of neutron flux in the civil work of a whole reactor

\section{Chaining Monte-Carlo simulations with external software}

As the result of the easy creation of large set of tallies on 3D structures with VICTORIA, the Monte Carlo results can be easily given as input data to external software. This section illustrates two applications of chaining calculations.

\subsection{Chaining Monte Carlo simulation results with finite elements analyses}

In this application VICTORIA is used to generate a MCNP input files for energy deposition (neutron and gamma) calculations on the reactor internals. This study case is the heavy neutron reflector surrounding the $\mathrm{EPR}^{\mathrm{TM}}$ core. The heavy neutron reflector (HNR) is a thick steel equipment drilled by cylindrical holes to allow the flow of water coolant. The challenge of this analysis is to calculate the energy deposition on the steel part and on a 3D user defined mesh. The geometry of the heavy neutron reflector is so built by a CAD import, then the part is sliced along a Cartesian pattern in order to define an energy deposition tally on each resulting piece of reflector. Figures 9 and 10 show this process until the integration within the whole MCNP model of the core.



Figure 9. Spatial decomposition of equipment for energy deposition calculations 


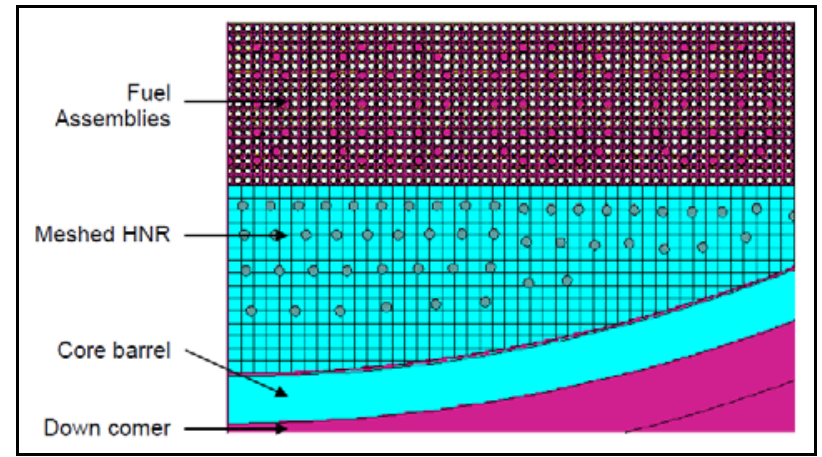

Figure 10. Integration of the meshed equipment in the MCNP model

The MCNP results of each tallies are then postprocessed to a CSV file containing the coordinates values of the center of each cells/tallies and the corresponding energy deposition value.

Such file format is readable by the main finite elements software packages to run thermomechanical analyses.

Such workflow is presented in figure 11 for the ANSYS Workbench software where the energy disposition is loaded as an external data of heat generation $\left(\mathrm{W} / \mathrm{cm}^{3}\right)$.

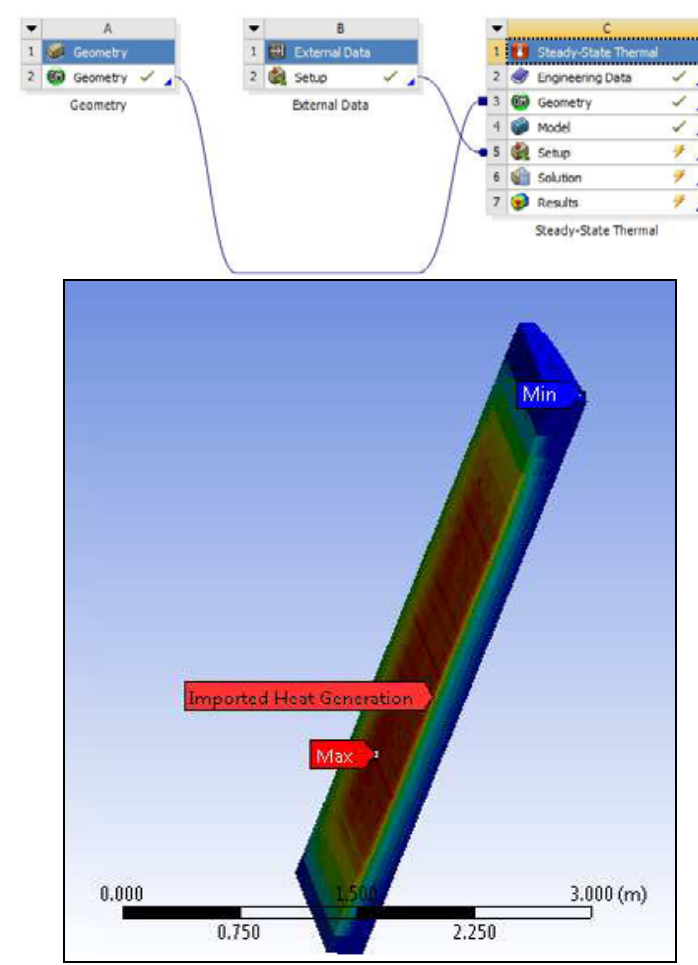

Figure 11. Load of MCNP results on a core internal for thermal steady state analysis

\subsection{Chaining with depletion codes for activation analyses}

Another kind of application requiring an efficient management of MCNP data results is the use of neutron spectrum calculations for activation assessment. In the context of the dismantling of reactors the prediction of activities inventory of structures surrounding the core is one of the key driver of the cost of the dismantling. Indeed the activities inventory is the input for waste classification assessment, doses evaluation leading to define the decommissioning scenario and schedule and related safety analyses. The AREVA NP methodology is based on Monte-Carlo 3D transport and is being used to support the dismantling of the French reactor fleet [7].

Once the neutron spectrum is calculated during power operation on a user defined mesh the data are post-processed to a VTK file format structure permitting storage and $3 \mathrm{D}$ visualization.

Based on the detailed material composition and power history an activation and depletion calculation (DARWIN or SCALE) is executed on each voxel. The results scalars fields are nuclide inventories, waste classification indicator, gamma source terms on user specified decay time steps.

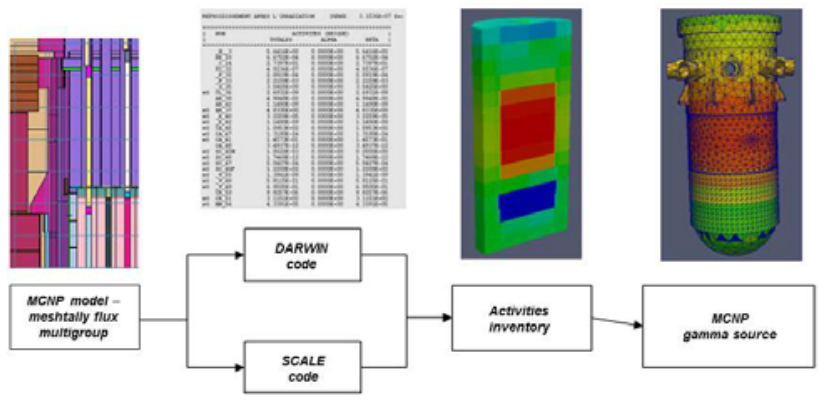

Figure 12. Illustration of the coupling between MCNP flux calculations and activation/depletion calculation codes

This chaining avoids human errors in the management of these huge quantities of data (storage of neutron spectrum and history, creation of depletion calculation code inputs, post-treatment of extraction of inventories quantities). The activation gradient can so be calculated on an adequate 3D mesh avoiding considering penalizing values for all the equipment/concrete solid. More generally having an automate workflow expedites modeling reliable and high fidelity evaluations.

\section{Conclusions}

This paper presents recent progress of AREVA developments. The suite of engineering tools has been drastically upgraded in order to offer Monte Carlo calculations as a reliable and efficient solution for radiation propagation projects. The main development is the VICTORIA software (Various Instances CAD calculation TOol and Radiation-propagation Interface by AREVA) offering CAD integration for both MCNP and TRIPOLI solvers. The calculations running times have been decreased by using modern technics. The use of Monte Carlo solvers is widespread from conceptual design phase $[5,6]$ to decommissioning [7] for any kind of reactor fleet or nuclear facility.

The versatility of the tools developed enables prompt answer to the needs as:

- New plant design and life extension analyses (Fluence, heating, sensors responses and 
embrittlement of equipment surrounding the core),

- Radiation protection studies and unpredicted radiological hazards,

- Support to Dismantling and Decommissioning for waste characterization, inventories and dose to operators calculations.

Monte Carlo methods are now much more affordable for projects avoiding simplifications and conservatisms of simpler methods. Monte-Carlo 3D with continuous energy analysis are superseding previous approaches where $2 \mathrm{D}$ models with multi group energy bins were applied.

Future developments are planned for the VICTORIA software to improve the user experience with new features.

\section{References}

1. F. Brown, B. Kiedrowski, J. Bull, "MCNP5-1.60 Release Notes", LA-UR-10-06235, (2010)

2. E. Brun, F. Damian, Eric Dumonteil, "TRIPOLI-4 version 8 User Guide, CEA-R-6316", (2013)

3. J. C. Wagner, E. D. Blakeman, D. E. Peplow, "Forward-weighted CADIS method for variance reduction of Monte Carlo calculations of distributions and multiple localized quantities", in International Conference on Mathematics, Computational Methods \& Reactor Physics - M\&C 2009, Saratoga Springs, N.Y., (2009)

4. Th. M. Evans et al. "Denovo: a new Threedimensional parallel discrete ordinates code in scale", Nuclear Technology vol. 171, (2010)

5. N. Chapoutier, A. Janet, G. Nolin, "ASTRID Core Shielding - Design Studies and Benchmark Analysis", Proceedings of ICAPP 2015, Paper 15305, (2015)

6. C. Jammes, N. Chapoutier, P. Filiatre, "Neutron flux monitoring system of the French GEN-IV SFR: assessment of diverse solutions for in-vessel detector installation", Nuclear Engineering and Design; v. 270, (2014)

7. M. Culioli, N. Chapoutier, S. Barbier, S. Janski, "State of the art of Monte Carlo technics for reliable activated waste evaluations", International Symposium on PREparation for DECommissionning, Lyon (2016) 